Commun. math. Phys. 32, 291-304 (1973)

(C) by Springer-Verlag 1973

\title{
Instability of Closed Spaces in General Relativity
}

\author{
Dieter R. Brill ${ }^{\star}$ \\ Max Planck Institute for Physics and Astrophysics, Munich, Germany \\ and
}

Department of Physics and Astronomy, University of Maryland, College Park, Md., USA

\section{Stanley Deser ${ }^{\star \star}$}

Laboratoire de Physique Théorique et Hautes Energies, Orsay, France

and

Physics Department, Brandeis University, Waltham, Mass., USA

Received March 7, 1973

\begin{abstract}
There are strong restrictions on the solutions of the initial value constraints of General Relativity when the spatial hypersurface is closed. In particular, closed flat space is unstable: not all solutions of the linearized constraints correspond to nearby solutions of the constraints themselves. For example, no nearby solutions whatever exist which are time symmetric. Other restrictions, which limit perturbations of non-flat closed initial solutions, are also exhibited.
\end{abstract}

\section{Introduction}

Although General Relativity may be characterized as a massless spin two Lorentz covariant field theory, its non-linearities can lead to qualitative complications not encountered in other field theoretical models. Most evident is the existence of solutions describing closed universes, in which the usual asymptotic falloff at infinity is absent. We shall investigate here some implications of the initial value equations peculiar to closed universes. The basic point is that flux integrals vanish identically when there is no boundary. In electrodynamics, for example, the total charge in a closed universe must be zero (because it can be expressed as a surface integral). The analogous gravitational quantity in asymptotically flat spaces is the total energy, which also can be expressed as a surface integral. The energy is due not only to sources but also to the gravitational field itself, and it has positive definiteness properties in its field dependence. Thus, if the mass is required to vanish, no excitations whatever are allowed. Below we investigate the restrictions which the

* Supported in part by the National Science Foundation and by the HumboldtFoundation.

$\star \star$ Supported in part by Grant AFOSR 70-1864. 
vanishing of energy-momentum surface integrals place on perturbations of closed spaces.

These restrictions are conveniently derived and discussed in terms of an orthogonal decomposition of perturbations into transverse and longitudinal parts. Here a new feature arises in the case of closed spaces, that additional variables beyond those familiar from asymptotically flat space are needed for a description of the general perturbation. For example, in a space of 3-torus topology, all "local" excitations may vanish (locally flat space), but in order to describe the space uniquely, some "global" variables (such as the total volume) still need to be specified.

We shall show that for the case of flat space with toroidal or other allowed [1] spatial topology, there are strong restrictions on allowed perturbations, which lead to instability of the solution. Instability is found in classes of solutions - e.g. time-symmetric flat universes which otherwise would appear to be a physically meaningful class of systems. For asymptotically flat spaces, stability means [2] that not only do the linearized equations permit geometries representing small perturbations, as is implicit in all local measurements, but that there are always rigorous solutions of the full equations "near" such varied spaces. In the case of general perturbations of flat closed space we find that the new variables of the orthogonal decomposition play an essential role. If they are taken to vanish, the space is completely isolated: there are no "neighbors" of flat space whose volume does not change in time at the initial surface. Even if the volume is allowed to change, strong restrictions are placed on the allowed perturbations ("instability"). For non-flat closed initial topologies we also find some unexpected restrictions on allowed perturbations ${ }^{1}$. We emphasize that our discussion of stability is entirely concerned with the initial value problem and does not involve the question of time-evolution, with eventual formation of singularities or collapse.

\section{Surface Integrals and Harmonic Functions}

The concept of total mass only makes physical or mathematical sense in asymptotically flat spaces, since mass is a constant of motion for isolated systems, defined $[3,4]$ by a surface integral over the system's boundary. A fortiori, the difference in "mass" $\delta m$ between two geometries on the same manifold,

$$
\delta m=\int d^{3} r \mathrm{~g}^{i j} \delta R_{i j}=\oint d S_{k}\left(\mathrm{~g}^{i j} \delta \Gamma_{i j}^{k}-\mathrm{g}^{i k} \delta \Gamma_{i j}^{j}\right)
$$

${ }^{1}$ After completing our work we received a preprint "Linearization stability of the Einstein equations" by A. Fischer and J. Marsden which also discusses the stability of the initial value problem on closed manifolds from a deeper mathematical point of view. 
is also a surface integral which must vanish for a closed space. On the other hand, the mass and its variation are also expressible as a volume integral of the geometry, e.g.

$$
\delta m=\int d^{3} r\left[-\delta \mathrm{g}^{i j}\left(R_{i j}+2 T_{i j}-T g_{i j}\right)+\pi_{i j} \delta \pi^{i j}-\frac{1}{2} \pi \delta \pi\right]
$$

so that its vanishing places restrictions on the allowed perturbations. Similar arguments hold for the other three components $\vec{P}$ of the energymomentum vector.

The integral relationship implied by the initial value equations can be obtained by using a systematic method, which we illustrate for the case of electromagnetism. To discover what restrictions must be placed on $\varrho$ in order that the covariant Gauss equation

$$
E_{\mid i}^{i}=\varrho
$$

have solutions we preceed as follows: (1) Find the appropriate decomposition of $E^{i}$ into transverse and longitudinal parts, in this case

$$
E^{i}=E^{i T}+\Phi^{\mid i}
$$

Here $E^{i T}$ satisfies $E^{i T}{ }_{\mid i}=0$ and $\Phi$ is a scalar potential; (2) Substitute (2.4) into (2.3) to obtain

$$
\nabla^{2} \Phi=\varrho .
$$

Thus to find $\Phi$ we must invert the self-adjoint operator $\nabla^{2}$; (3) Use the theorem that a self-adjoint operator can be inverted if the source has no component along any of the harmonic functions $H$ of the operator, which satisfy (in this case)

$$
\nabla^{2} H=0 .
$$

In open spaces with boundary conditions $H=0$ at infinity, the only harmonic function is $H=0$, while in a closed space, $H=$ const is also allowed. Hence from (2.5) we find the condition

$$
\int H \varrho d V=H \int \varrho d V=0
$$

which implies the usual result of a vanishing total charge, obtained here in a systematic way which can be generalized to the case of gravitation.

\section{Orthogonal Decomposition in a Closed Space}

To apply the above method to a perturbation $\delta g_{i j}, \delta \pi^{i j}$ of a closed space, we first investigate the "harmonic" tensor functions of the operators involved in a decomposition of tensors into transverse and longitudinal 
parts according to [5]

$$
h_{i j}=h_{i j}^{t}+\left(h_{i \mid j}+h_{j \mid i}\right)=h_{i j}^{t}+h_{(i \mid j)} .
$$

Here $h_{i j}^{t}$ is to satisfy the transversality condition $h_{i \mid j}^{t j}=0(\mid$ denotes covariant differentiation in the background geometry). We take the divergence of (3.1) to find

$$
h^{i j}{ }_{\mid j}=h^{(i \mid j)}{ }_{\mid j} .
$$

The right side of (3.2) represents a self-adjoint operator acting on the vector $h^{i}$, and the left side is the source analogous to the $\varrho$ of Eq. (2.3). To find the "harmonic" vectors $k^{i}$ of this operator,

$$
k^{(i \mid j)}{ }_{\mid j}=0
$$

we multiply Eq. (3.3) by $k_{i}$ and integrate over the whole space,

$$
\begin{aligned}
0 & =\int k_{i} k^{(i \mid j)}{ }_{\mid j} d V=-\int k_{i \mid j} k^{(i \mid j)} d V \\
& =\frac{1}{2} \int k_{(i \mid j)} k^{(i \mid j)} d V .
\end{aligned}
$$

Due to the quadratic nature of the integrand in (3.4) we must have

$$
k^{(i \mid j)}=0
$$

for these vectors $k^{i}$ : the harmonic vectors of the operator of Eq. (3.2) are Killing vectors. Thus the condition of integrability of (3.2) is that the source be orthogonal to any Killing vectors of the space. But this is guaranteed by the structure of the source and by Eq. (3.5):

$$
\int k_{i} h^{i j}{ }_{\mid j} d V=-\int k_{i \mid j} h^{i j} d V=-\frac{1}{2} \int k_{(i \mid j)} h^{i j} d V .
$$

Thus the decomposition (3.1) is always possible; if there are Killing vectors, $h_{i}$ is determined only up to such Killing vectors, but the longitudinal part $h_{i \mid j}+h_{j \mid i}$ is uniquely determined.

The transverse part $h_{i j}^{t}$ can be decomposed into a transverse traceless part $h_{i j}^{T T}$ and a trace $h_{i j}^{T}$. Usually the trace is written as the transverse operator acting on the scalar trace $h^{T}$,

$$
h_{i j}^{t}=h_{i j}^{T T}+\frac{1}{2}\left(g_{i j} \nabla^{2}-\nabla_{i} \nabla_{j}\right) h^{T} .
$$

Then $h^{T}$ is determined by the trace of (3.7),

$$
h_{k}^{t}{ }_{k}=\nabla^{2} h^{T}
$$

which, as before, can be solved only if the source $h_{k}^{t k}$ is orthogonal to all harmonic scalars (i.e., constants). Since this is in general not the case, the decomposition (3.7) must be supplemented by the component of $h_{k}^{t}{ }_{k}$ along the constants,

$$
V^{-1} \int h_{k}^{t} k d V \equiv c_{h}
$$


(where $V$ denotes the total volume of the 3 -space). The complete decomposition therefore takes the form

$$
h_{i j}=h_{i j}^{T T}+\frac{1}{2}\left(g_{i j} \nabla^{2}-\nabla_{i} \nabla_{j}\right) h^{T}+\frac{1}{3} g_{i j} c_{h}+h_{(i \mid j)} .
$$

The term in $c_{h}$ does not appear in the decomposition of a tensor (with vanishing boundary conditions) in flat Euclidean space. Thus, besides the usual irreducible tensor, vector and scalar fields, the decomposition of a tensor in a closed manifold requires one additional number which describes a "global" property of the tensor, appropriate to this topology.

\section{Solution of the Varied Constraints}

The gravitational field may be characterized in terms of the initial 3-geometry $\left(g_{i j}\right)$ and extrinsic curvature $\left(\pi^{i j}\right)$ of some spacelike surface, subject to the four initial value equations (constraints). We investigate the conditions under which a perturbed geometry $\left(g_{i j}+\delta g_{i j}, \pi^{i j}+\delta \pi^{i j}\right)$ satisfies the varied constraints if $\left(g_{i j}, \pi^{i j}\right)$ is a solution. In the absence of sources, the constraint equations are

$$
\begin{gathered}
\mathfrak{R}-\mathfrak{I}=0 \\
\pi^{i j}{ }_{\mid j}=0 \\
\mathfrak{I} \equiv g^{-\frac{1}{2}}\left(\pi_{i j} \pi^{i j}-\frac{1}{2} \pi_{i}^{i 2}\right)
\end{gathered}
$$

where $\mathfrak{R}$ is the three-dimensional curvature scalar density corresponding to $g_{i j}$, and a bar again denotes covariant differentiation with respect to the spatial metric. For simplicity in treating the nonlinear constraints we expand them to various orders in the varied metric. Each order must then separately vanish.

In first order the varied geometry must obey four (linearized) constraints, so that not all the components $\delta g_{i j}, \delta \pi^{i j}$ can be independent, four of them being determined in terms of the rest. The orthogonal decomposition (4.1) is appropriate to identify the independent and the constraint variables, and to determine the latter in terms of the former by the constraint equations. In the particular case of a flat closed space, the simplest unperturbed geometry (one allowing an extremal surface $\pi=0)$ is described by [4]

$$
R_{i j}=0, \quad \pi^{i j}=0 .
$$

In that case the first order constraints reduce to

$$
\delta R=g^{i j} \delta R_{i j}=0, \quad\left(\delta \pi^{i j}\right)_{\mid j}=0 .
$$


We can employ Cartesian coordinates $g_{i j}=\delta_{i j}$ on the surface, so that $\delta R_{i j}$ has the simple form

$$
2 \delta R_{i j}=-\nabla^{2} \delta g_{i j}^{T T}-\frac{1}{2}\left(g_{i j} \nabla^{2}+\nabla_{i} \nabla_{j}\right) \nabla^{2} \delta g^{T} .
$$

Thus we obtain

$$
\begin{gathered}
\delta R=-\nabla^{2} \nabla^{2} \delta g^{T} \\
\left(\delta \pi^{i j}\right)_{\mid j}=\delta \pi^{(i \mid j)}{ }_{\mid j}=0 .
\end{gathered}
$$

By the arguments of Section III we readily find from (4.7)

$$
\delta g=\text { const } .
$$

Similarly, from (4.8) and the argument leading to Eq. (3.5) we have

$$
\delta \pi^{(i \mid j)}=0 .
$$

Thus without loss of generality we may set $\delta g^{T}=0=\delta \pi^{i}$. The general solution of the first order constraint equations is then given by

$$
\begin{aligned}
\delta g_{i j} & =\delta g_{i j}^{T T}+\delta g_{(i \mid j)}+\frac{1}{3} g_{i j} c_{g} \\
\delta R_{i j} & =-\frac{1}{2} \nabla^{2} \delta g_{i j}^{T T} \\
\delta \pi^{i j} & =\delta \pi^{i j T T}+\frac{1}{2}\left(g^{i j} \nabla^{2}-\nabla^{i} \nabla^{j}\right) \delta \pi^{T}+\frac{1}{3} g^{i j} c_{\pi}
\end{aligned}
$$

where $c_{g}, c_{\pi}$ are constants.

Physically the allowed unconstrained variations correspond to two pairs of dynamical excitations $\left(\delta g^{T T}, \delta \pi^{T T}\right)$, and two sets of coordinate variations $\left(\delta g_{i}, \delta \pi^{T}\right)$, exactly as in open topologies, together with the new pair of constants $\left(c_{g}, c_{\pi}\right)$. To first order, then, there are no new restrictions in closed spaces (in open spaces there is the further integral consequence [4] that $\delta m$ vanishes as a result of (4.11)).

The new variables $c_{g}, c_{\pi}$ have a clear physical meaning for closed spaces: $c_{g}$ measures the perturbation of the total volume (assuming that the perturbed and unperturbed geometries are described by the same atlas, or by coordinates with the same range),

$$
c_{g}=2 \delta \mathrm{V} / \mathrm{V} \text {. }
$$

Similarly ${ }^{2}, c_{\pi}$ describes the time rate of change of the (perturbed) total volume $V$ in normal geodesic coordinates (lapse $N=1$, shift $N^{i}=0$ ):

$$
c_{\pi}=d\left(\ln \delta V^{-2}\right) / d t .
$$

${ }^{2}$ Such properties as the total volume and its time rate of change are not the only global variables peculiar to closed spaces; for example, a three-torus without curvature excitation (flat space) is characterized by five global variables in addition to the total volume (for example, a variation of the dimensions of the torus keeping the volume constant). These additional global variables do not need new terms in the orthogonal decomposition for their description, but correspond to harmonic transverse traceless perturbations. Clearly, variation of these variables does not affect the constraints. 
The coordinate variations can be fixed by coordinate conditions: $\delta g_{i}$ are determined if conditions are given on the coordinates within the initial surface, and a determination of $\delta \pi^{T}$, as usual, amounts to fixing the initial surface itself within the embedding 4-space. In open spaces a suitable condition is the "extremal surface" condition,

$$
\delta \pi^{T}=0 .
$$

This condition can also be used in closed space (even though extremal surfaces are atypical in such spaces) because the additional terms in (4.11) imply that in this case (4.14) describes surfaces of constant (rather than vanishing) extrinsic curvature,

$$
g_{i j} \delta \pi^{i j}=c_{\pi} .
$$

Thus with coordinate condition (4.14), $c_{\pi}$ plays the role of time in the varied geometry.

\section{Second Variation of Constraints Near Flat Space}

We now turn to the second order terms in the timelike constraint, $\delta^{2}(\mathfrak{R}-\mathfrak{I})=0$, subject of course to the relations among the variations implied by $\delta(\mathfrak{R}-\mathfrak{I})=0$. In a linear theory the second order equations never restrict the first order variables, since they can always be solved by setting the second order variations equal to zero. In a nonlinear theory, the second order integrability conditions may involve the first order variations quadratically; but if the first order integrability conditions are all nontrivial, but can be satisfied by restricting some variables, then the second order conditions can be satisfied by restricting the same variables to second order (see Section VIII). Here we are interested in the opposite case, where the first order condition is identically satisfied. A nontrivial condition may then appear in second order, and signal instability. This case corresponds to $R_{i j}=0=\pi^{i j}$ (cf. Eq. (8.5)). Even here there is no problem in open spaces [4] in satisfying the timelike constraint. However, in the case of closed 3-space we shall see that the second order timelike constraint can only be satisfied if the first order variations satisfy, in addition to $\delta(\mathfrak{R}-\mathfrak{I})=0, \delta\left(\pi^{i j}{ }_{j j}\right)=0$, a strong additional integrability condition, which leads to instability.

Using $\mathfrak{R}=\mathfrak{g}^{i j} R_{i j}$, the expression (4.3) for $\mathfrak{T}$, and $R_{i j}=0=\pi^{i j}$ for the unperturbed geometry we find

$$
0=\delta^{2}(\mathfrak{R}-\mathfrak{T})=\delta \mathfrak{g}^{i j} \delta R_{i j}+\delta\left(\mathfrak{g}^{i j} \delta R_{i j}\right)-\delta \pi_{i j} \delta \pi^{i j}+\frac{1}{2}\left(\delta \pi_{i}^{i}\right)^{2} .
$$

Only the second term contains the $\delta^{2} g_{i j}$ (through $\mathrm{g}^{i j} \delta^{2} R_{i j}$ ), and all other terms are to be regarded as its source. By the Palatini identity 
$\left(\delta R_{i j} \equiv \delta \Gamma^{k}{ }_{i j \mid k}-\delta \Gamma_{i k \mid j}^{k}\right)$ this second term is a divergence, and if the decomposition (3.10) for $\delta^{2} g_{i j}$ is substituted into it we find,

$$
\delta\left(\mathrm{g}^{i j} \delta R_{i j}\right)=(\text { divergence })-g^{\frac{1}{2}} \nabla^{2} \nabla^{2} \delta^{2} g^{T}
$$

where we have denoted all the terms not involving $\delta^{2} g_{i j}$ by (divergence), and all covariant operators are evaluated in the unperturbed geometry in Cartesian coordinates.

Now we again use the condition for existence of a solution $\delta^{2} g^{T}$, namely that the source must be orthogonal to the harmonic functions of $\nabla^{2}$ (i.e., constants), so that we must have the integrability condition

$$
\int\left[\delta \mathrm{g}^{i j} \delta R_{i j}-\delta \pi_{i j} \delta \pi^{i j}+\frac{1}{2}\left(\delta \pi_{i}^{i}\right)^{2}\right] d^{3} x=0 .
$$

This can be simplified by substituting the orthogonal decomposition (4.11) and its expression for $\delta R_{i j}$, and integrating by parts to find

$$
\begin{gathered}
\int\left(g^{m n} \delta g_{i j, m}^{T T} \delta g_{k l, n}^{T T}+\delta \pi_{i j}^{T T} \delta \pi_{k l}^{T T}\right) g^{i k} g^{j l} g^{\frac{1}{2}} d^{3} x \\
=\frac{1}{6} \int c_{\pi}^{2} g^{\frac{1}{2}} d^{3} x=V c_{\pi}^{2} / 6 .
\end{gathered}
$$

This condition can be taken as an equation determining $c_{\pi}$. This quantity however has been shown to be a degree of freedom on a par with the usual two degrees of freedom per space point, $\delta g^{T T}, \delta \pi^{T T}$ : this was the consequence of the orthogonal decomposition and analysis of first variation. Thus Eq. (5.4) is a constraint on one of the dynamical variables of the system.

The additional restriction (5.4) on the dynamical variables means that the manifold of solutions is smaller than the "tangent space" defined by the first order constraints, i.e. that the initial value problem is unstable: there is no solution for a general set of allowed first order perturbations of the initial data $\left(\delta g^{T T}, \delta \pi^{T T}, c_{g}\right.$ and $\left.c_{\pi}\right)$ because this general set will not satisfy (5.4). Even stronger instability follows if we require $c_{\pi}=0$ (which means that the same time choice parametrizes the unperturbed and perturbed spacelike surface; cf. Eq. 4.15). It then follows from (5.4) that flat space is the only solution [6]. This is so because the left side of (5.4) is positive definite, so that it can only vanish if $\delta g_{i j}^{T T}=$ const and $\delta \pi_{i j}^{T T}=0$, hence $\delta R_{i j}=0$, and (using (4.12) and $\left.c_{\pi}=0\right) \delta \pi_{i j}=0$. Thus, the initial value problem in a flat closed manifold is always maximally unstable if the initial and varied 3-geometries are extremal surfaces. One might try to argue that there is stability in the above situation, in the sense that $c_{\pi}$ replaces $\delta m$ as "dependent variation" determined by the other constraints, so that its value is such as to make (5.4) valid for arbitrary $\left(\delta g^{T T}, \delta \pi^{T T}\right)$. This is not the case: $\delta m$ is indeed an auxiliary quantity, entirely determined by the $(\delta g, \delta \pi)$ and not involved in the varied constraints themselves (it is zero at flat space, for example). Likewise, $\delta^{2} m$ is deter- 
mined to be equal to the left side of (5.4). On the other hand, $c_{\pi}$ (along with other components) is left free by the first variations, but it is one of the excitation variables. The instability lies in its then being forced to be a fixed functional of the other variations by the second variation equations, namely to obey (5.4). Thus $c_{\pi}$ is on an altogether different footing from $\delta m^{3}$

One can easily convince oneself that this situation repeats in higher orders beyond the second, but that no further conditions on the first order perturbations are implied by higher variations. One may also easily generalize the above derivation to include dynamical, positive matter sources $T_{\mu v}$. Effectively, $\mathfrak{T}^{0}{ }_{0}$ of any physical matter source will have near vacuum a positive bilinear form analogous to that of the "source" $\delta \pi_{i j} \delta \pi^{i j}$ and will be excluded by the second variation in a similar way. This excludes e.g. addition of slight amounts of matter to the extremal surface closed vacuum state.

\section{Integrability of the Spacelike Constraints}

In closed spaces with Killing vectors, the three spacelike constraints (4.2) are another source of instability, as first shown by Wald and Geroch [8]. In this section we analyze these constraints by the methods of Section II. For a general background geometry the first variation of the constraints contains terms in $\delta \pi^{i j}$ and $\delta g_{i j}$ (the latter due to the change in the covariant derivative). When we substitute for the density $\delta \pi^{i j}$ a decomposition of type (3.10) we again find an equation of the Poisson type,

$$
\delta \pi^{(i \mid j)}{ }_{\mid j}=-\delta \Gamma_{j k}^{i} \pi^{j k} .
$$

The operator on the left was discussed in Section III, and was shown to have no "harmonic" functions (Killing vectors) in general closed space. Thus in general the spacelike constraints impose no first order integrability conditions on the perturbations. This is obvious when there are no Killing vectors, and also at flat space where the right side vanishes. One must therefore examine the constraints to second order.

Killing vectors $k^{i}$ do exist in all closed flat topologies [1] that are of particular interest here. In this case the solution of the first order equations is given by Eq. (4.11), but integrability conditions follow from the second order equations,

$$
\delta^{2} \pi^{(i \mid j)}{ }_{\mid j}=-2 \delta \Gamma_{j k}^{i} \delta \pi^{j k} \equiv s^{i}
$$

${ }^{3}$ Also in other respects, $c_{\pi}$ plays quite a different role from $\delta m$; for example, for given $\delta g_{i j}^{T T}, \delta \pi_{i j}^{T T}$, and $c_{g}$, two values of $c_{\pi}, c_{\pi}= \pm\left|c_{\pi}\right|$ are possible. These new features peculiar to closed spaces are still largely unexplored. 
whose source $s^{i}$ must be orthogonal to all Killing vectors,

$$
\int k_{i} s^{i} d^{3} x=0 \text {. }
$$

This equation was found as a necessary condition by Wald and Geroch [8]. To simplify it we substitute the first order solution (4.11), integrate by parts, and note that, in a closed flat space, the Killing vectors describe infinitesimal translations but not rotations; therefore they satisfy a stronger equation than (3.5), namely $k_{i \mid j}=0$. The result is the relation

$$
\int k^{i} \delta g_{j k \mid i} \delta \pi^{j k} d^{3} x=\int\left(\mathscr{L}_{\boldsymbol{k}} \delta g_{i j}^{T T}\right) \delta \pi^{i j T T} d^{3} x=0
$$

which must be satisfied by the first order perturbations to make the second order constraints integrable. The existence of (6.4) indicates a further instability of the initial value solutions. (The timelike constraint left the " $T T$ " variables entirely unconstrained to first order, and the second order timelike integrability condition (5.4) in no way insures that (6.4) is satisfied.)

The equation corresponding to (6.4) in open spaces would have a right hand side proportional to the total momentum surface integral, to second order; it can therefore always be satisfied for arbitrary $\delta g^{T T}$, $\delta \pi^{T T}$ by proper asymptotic dependence. The present instability can be physically understood as a condition of vanishing total momentum in closed spaces, analogous to the view of Eq. (5.4) as a condition of vanishing total energy. (In both cases of course the first variations vanish about flat space.)

Eq. (6.4) may be rewritten in the form

$$
\int k_{(i ; j)} \delta \pi^{i j T T} d^{3} x=0
$$

where; denotes the covariant derivative in the perturbed metric $g_{i j}+\delta g_{i j}$. Thus $\delta \pi^{i j T T}$ must be orthogonal to the tensor $k_{(i ; j)}$ in the perturbed geometry. This suggests as the reason for the instability the existence of "extra" $T T$ tensors [7] $\mathscr{L}_{\boldsymbol{k}} \delta g_{i j}^{T T}$ which solve the linearized spacelike constraints, but which are longitudinal $\left(k_{(i ; j)}\right)$ in the perturbed geometry, and therefore cannot be expected to be unconstrained. The relation of these "extra" $T T$ tensors to the total momentum integrability condition (6.4) is similar to that of the new variable $c_{\pi}$ to the energy integrability condition (5.4): in each case the condition goes hand in hand with existence of new variables which allow the condition to be satisfied.

\section{Particular Perturbations of a Closed Flat Space}

We have seen that closed flat space is isolated if we demand $c_{\pi}=0$, and unstable if we allow $c_{\pi} \neq 0$. In order to show that the instability 
cannot be strengthened to isolation also for $c_{\pi} \neq 0$, we show how to construct a continuous family of exact solutions which includes the flat 3 -torus in one limit. Such a family is possible because, unlike in asymptotically flat space, one cannot assume that a coordinate condition on $\pi$ can always be implemented. In particular, none of the family of solutions except the unperturbed 3-torus can have any spacelike surface on which $c_{\pi}=0$.

From any translation-homogeneous space of Euclidean topology one can construct a 3-torus topology by forming the quotient space with a discrete group of three independent translations. The family of Kasner metrics [9]

$$
\begin{gathered}
d s^{2}=-d t^{2}+t^{2 p} d x^{2}+t^{2 q} d y^{2}+t^{2 s} d z^{2} \\
p+q+s=1 \quad p^{2}+q^{2}+s^{2}=1
\end{gathered}
$$

provides examples of solutions with the necessary homogeneity. The corresponding toroidal solutions contain flat 4-space as a special case, e.g. $p=1, q=s=0$. However, there is no choice of spacelike surface in this geometry which describes the flat 4-space or its perturbations in terms of a flat and plane initial surface. (This is the 4-dimensional analog of the flat surface of an ordinary cone, on which there are likewise no everywhere straight slices.) Instead, in order to construct a family which contains the flat 3-torus as a limit we fix the values of $p, q$, and $s$, and choose, for each $T$, translations of the type

$$
x \rightarrow x+n T^{-p} \quad y \rightarrow y+m T^{-q} \quad z \rightarrow z+l T^{-s}
$$

with $n, m, l$ integers. The spacelike surfaces $t=T$ in the corresponding family of quotient spaces become flat in the limit $T \rightarrow \infty$, because the curvature of these toroidal spacelike surfaces decreases as $T^{-2}$, and their size is independent of $T$ due to the identification (7.2). At finite $T$ the spacelike surfaces are non-flat, and therefore show the existence of quite nontrivial perturbations of the initial value problem in the closed flat torus ${ }^{4}$. In accordance with the theorem of Section V, there can be no spacelike surface in these solutions with $d V / d t=0$. Instead, the total volume continues to expand, and a recontraction phase is never possible.

For other topologies such as the Friedmann or the sourcefree Taub universe [11] the restriction (5.4) is not directly relevant, since an integrability condition arises already in first order. These conditions are examined (for the sourcefree case) in the next section.

${ }^{4}$ Inhomogeneous spaces of toroidal topology have been recently discussed by Gowdy [10]. Other classes of non-flat solutions have recently been given by $\cdot Y$. ChoquetBruhat [13]. 


\section{Closed Non-Flat Spaces}

At flat space, additional restrictions on the varied initial data were imposed by the vanishing of the second variations of the surface integrals $\left(\delta^{2} m, \delta^{2} P\right)$, their first variations vanishing identically. We now consider arbitrary non-flat initial geometries. A first example is furnished by an arbitrary 3-geometry $\left(R_{i j} \neq 0\right)$ at a moment of time symmetry $\left(\pi^{i j}=0\right)$. Variation of the time-like constraint yields

$$
0=\delta \Re=R_{i j} \delta \mathrm{g}^{i j}+\mathrm{g}^{i j} \delta R_{i j}=\delta \mathrm{g}^{i j} R_{i j}+\text { div } .
$$

This equation may be rewritten as a Poisson equation for the trace part, $g^{i j} \delta g_{i j}$, of the variation, whose source is proportional to the traceless part [4]. But (8.1) does more than constrain this trace component, since its integral immediately implies orthogonality of the full metric variations and the curvature:

$$
0=\int d^{3} r \delta \mathrm{g}^{i j} R_{i j}
$$

On the other hand, the Bianchi identity, $\nabla_{j}\left(R^{i j}-\frac{1}{2} g^{i j} R\right) \equiv 0$, together with the constraint, $R=0$, imply that $R_{i j}$ is transverse traceless. Since every $T T$ tensor is identically orthogonal to any non- $T T$ one, we may write (8.2) as

$$
0=\int d^{3} r \delta \mathrm{g}^{i j T T} R_{i j}
$$

This states that the otherwise unconstrained pure spin 2 variations $\delta g^{T T}$ must be orthogonal to the existing excitations of the base geometry.

The apparent "linear instabilities" implied by (8.3) or (8.5) below are not really instabilities, however. Rather, these equations are simply part of the conditions, imposed by the varied constraints themselves, defining the tangent space of allowed perturbed geometries. They only appear to be restrictions when formally compared with the flat case, or with the asymptotically flat problem. But it is simply an integral part of the constraints in closed topologies that they both specify a constraint variable and require the vanishing of the "monopole moment" of its source. The same remarks apply to (8.6), that it is just part of the second variation, $\delta^{2}(\mathfrak{R}-\mathfrak{I})=0$ of the constraint.

For the general case, $\pi^{i j} \neq 0 \neq R_{i j}$, (8.1) becomes

$$
0=\delta(\mathfrak{R}-\mathfrak{I})=\delta \mathfrak{g}^{i j}\left(R_{i j}+2 T_{i j}-T g_{i j}\right)-\delta \pi^{i j}\left(\pi_{i j}-\frac{1}{2} g_{i j} \pi\right)+\operatorname{div}
$$

and can be shown to determine the same constraint variable as (8.1). The corresponding integral requirement is

$$
0=\int d^{3} r\left[-\delta g^{i j}\left(R_{i j}+2 T_{i j}-T g_{i j}\right)+\delta \pi^{i j}\left(\pi_{i j}-\frac{1}{2} g_{i j} \pi\right)\right],
$$


and again involves the unconstrained components of the first variations $\left(\delta g^{i j}, \delta \pi^{i j}\right)$.

At second order, there will also be an integrability condition as a generalization of (5.3). Again, for simplicity, consider the case $\pi^{i j}=0$, where variation of $(8.5)$ yields

$\int d^{3} r\left(\delta^{2} \mathrm{~g}^{i j}\right) R_{i j}=-\int d^{3} r\left[\delta \mathrm{g}^{i j} \delta R_{i j}+\delta \pi^{i j} \delta \pi^{l m}\left(g_{i l} g_{j m}-\frac{1}{2} g_{i j} g_{l m}\right) g^{-\frac{1}{2}}\right]$.

At flat space, the left side vanishes and this reduces to (5.3) as a condition on the first variations. When $R_{i j} \neq 0$, however, it becomes a restriction, analogous to (8.3), on the $T T$ part of $\delta^{2} g^{i j}$ (since $R_{i j}$ is $T T$ here) in terms of the first variations. Its character is therefore quite different from that of (5.4) which restricted first variations only; it does not affect stability about the base space.

Finally, we examine the spacelike constraints. As discussed in VI, no conditions are imposed by their variations in the absence of Killing vectors. Consider, e.g., (6.1),

$$
\nabla_{j}\left(\delta \pi^{i j}\right)=-\delta \Gamma^{i}{ }_{l j} \pi^{j l} \equiv \varrho^{i} .
$$

Since only integrals of scalar densities are meaningful, we only obtain an interesting condition if there is a "constant" vector present, namely a translational Killing vector $\left(k_{i \mid j}=0\right)$. [In open topology, the Killing vectors of the asymptotic Minkowski space are available.] In such exceptional geometries, we obtain the condition

$$
0=\int d^{3} r k^{i} \nabla_{i}\left(\delta g_{l m}\right) \pi^{l m}=-\int d^{3} r k^{i} \delta g_{l m} \nabla_{i} \pi^{l m}
$$

as a linear restriction on the allowed $\delta g_{l m}$ about spaces admitting a $k_{i}$. Similar remarks apply to the second variations, which also only yield conditions when $k_{i}$ are present. Even then, however, it is clear from the foregoing discussion that (8.8) is not a new condition but part of the varied constraint (8.7). We conclude that instability is a phenomenon peculiar to closed flat space.

\section{Open Geometries}

For asymptotically flat open topologies, surface integrals no longer vanish, and in particular $m, \delta m, \delta^{2} m$ are now generally non-null flux integrals. We emphasize that their vanishing for closed spaces is related to the fact that the notion of energy is neither mathematically defined nor physically meaningful: Energy is related to the generator of time translation and is observed in terms of interaction among two (almost) isolated bounded systems. 
Because the formerly vanishing integrals now represent the variation of mass implied by a given perturbation, the restrictions we found in the closed case disappear. Indeed, there are both many solutions near open flat space and many allowed perturbed geometries as well and they agree in content, so that the open case is stable [2]. However, one may ask what restrictions are imposed on perturbations of the geometry if one arbitrarily imposes specific conditions on the perturbed energy (the $c_{g}, c_{\pi}$ are of course absent here). Thus, if the perturbation is a "rearrangement," leaving the total mass unchanged, then $\delta m=0=\delta^{2} m$, and we have results similar to the closed space case: If the unperturbed state is flat space, then since $\delta^{2} m$ is positive definite there [4], its vanishing eliminates all nearby spaces, i.e. there are no zero mass states near flat space. The restrictions implied by a rearrangement of a more general base geometry are of the same type as those found in Section VIII.

\section{References}

1. Nowacki, W.: Commentarii Mathematici Helvetici 7, 81 (1935).

2. Choquet-Bruhat, Y., Deser, S.: On Stability of Flat Space, Ann. Phys. (N. Y.) (to appear 1973).

3. Arnowitt, R., Deser, S., Misner, C.: Phys. Rev. 122, 997 (1961).

4. Brill, D., Deser, S.: Ann. Phys. (N. Y.) 50, 548 (1968).

5. Deser, S.: Ann. Inst. H. Poincaré 7, 149 (1967).

6. Brill,D.: Isolated Solutions in General Relativity, in Gravitation: Problems and Prospects, Naukova Dumka (Kiev 1972), p. 17.

7. York, J.: J. Math. Phys. 14, 456 (1973).

8. Wald, R., Geroch, R.: unpublished.

9. Kasner, E.: Am. Jour. Math. 43, 217 (1921).

10. Gowdy, R.H.: Phys. Rev. Letters 27, 826 (1971).

11. Taub, A. H.: Ann. Math. 53, 472 (1951).

12. Fischer, A.E., Marsden, J.E.: Linearization Stability of the Einstein Equations. Bull. Am. Math. Soc., September 1973

13. Choquet-Bruhat, Y.: Commun. math. Phys. 21, 211 (1971).

Dieter Brill

Max Planck-Institut

D-8000 München 23

Föhringer Ring 6

Federal Republic of Germany 